\title{
Four Cases of Pneumatosis Cystoides Intestinalis Complicated by Connective Tissue Diseases
}

\author{
Eiji Suzuki ${ }^{1,2}$, Takashi Kanno ${ }^{1}$, Momoko Hazama ${ }^{1}$, Hiroko Kobayashi ${ }^{2}$, \\ Hiroshi Watanabe ${ }^{2}$ and Hiromasa Ohira ${ }^{2}$
}

\begin{abstract}
Pneumatosis cystoides intestinalis (PCI) is a rare disease that involves the presence of gas in the intestinal wall. Connective tissue disease (CTD) is a major cause of secondary PCI. In addition to the nature of CTDs, the use of prednisolone and some immunosuppressants, and the presence of complicating diseases such as diabetes mellitus, constipation and pulmonary diseases are involved in the development of PCI. This report describes four cases of PCI with different CTDs (granulomatosis with polyangiitis, rheumatoid arthritis, dermatomyositis, and overlap syndrome) and discusses the background of each patient and common risk factors for the occurrence of PCI.
\end{abstract}

Key words: alpha glucosidase inhibitor, connective tissue disease, pneumatosis cystoides intestinalis, prednisolone

(Intern Med 56: 1101-1106, 2017)

(DOI: 10.2169/internalmedicine.56.7877)

\begin{abstract}
Introduction
Pneumatosis cystoides intestinalis (PCI) is a rare disease characterized by gas within the intestinal submucosa or subserosa. PCI is mainly classified into primary and secondary forms, with the latter resulting from conditions such as pulmonary diseases, gastrointestinal diseases, and traumatic injury. Connective tissue disease (CTD) represents an important background factor for secondary PCI (1-12). Furthermore, drugs such as $\alpha$-glucosidase inhibitors ( $\alpha$-GI) (13-15) and immunosuppressants (16) have been reported to drive the pathophysiology of PCI. However, the pathogenetic mechanisms underlying PCI have not been elucidated, although several hypotheses have been proposed. Why CTD patients are likely to show complications of PCI has yet to be clearly shown. We herein report four cases of PCI associated with granulomatosis with polyangiitis (GPA), rheumatoid arthritis (RA), dermatomyositis (DM), and overlap syndrome.
\end{abstract}

Case Reports

\section{Case 1}

A 71-year-old woman was admitted to our hospital with right nose pain. One year earlier, she had been diagnosed with GPA [sinusitis, lung nodules, and positive results for proteinase 3 anti-neutrophil cytoplasmic antibody (PR3ANCA)] and was treated with prednisolone (PSL) at $30 \mathrm{mg} /$ day. After treatment, the clinical symptoms disappeared. However, diabetes mellitus arose as a complication, so voglibose was added and the PSL dose was reduced. On admission, her height and weight were $149 \mathrm{~cm}$ and $56.4 \mathrm{~kg}$, respectively, and her body temperature was $35.9^{\circ} \mathrm{C}$. A clinical examination showed right malar rash, saddle nose, and right abdominal distension, but no tenderness. The laboratory findings on admission were as follows: white blood cell (WBC) count, 9,400/ $\mathrm{L}$ (neutrophils, 83.4\%; monocytes, 4.4\%; lymphocytes, 11.7\%); hemoglobin ( $\mathrm{Hb}), 11.5 \mathrm{~g} / \mathrm{dL}$; platelet count, $31.7 \times 10^{4} / \mu \mathrm{L}$; total protein, $6.2 \mathrm{~g} / \mathrm{dL}$; albumin, $3.5 \mathrm{~g} / \mathrm{dL}$; lactic dehydrogenase (LDH), $391 \mathrm{U} / \mathrm{L}$; creatine

\footnotetext{
${ }^{1}$ Department of Rheumatology, Ohta-Nishinouchi Hospital, Japan and ${ }^{2}$ Department of Gastroenterology and Rheumatology, Division of Medicine, Fukushima Medical University School of Medicine, Japan
}

Received for publication June 18, 2016; Accepted for publication August 21, 2016

Correspondence to Dr. Eiji Suzuki, azsuzuki@ohta-hp.or.jp 
(a)

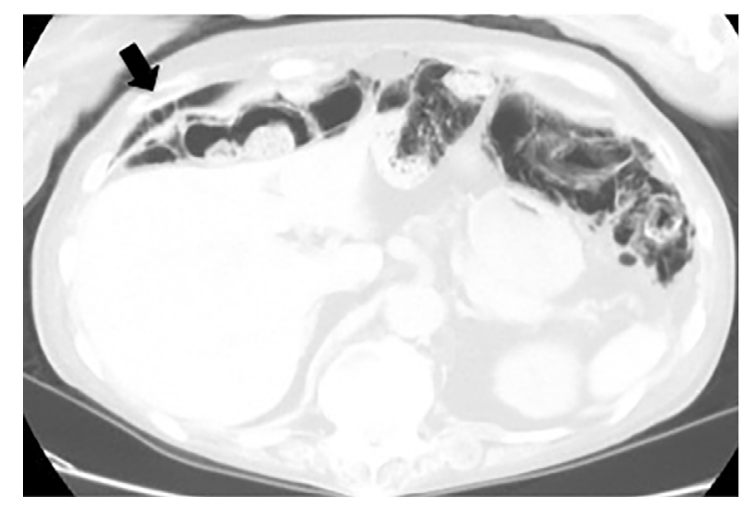

(b)

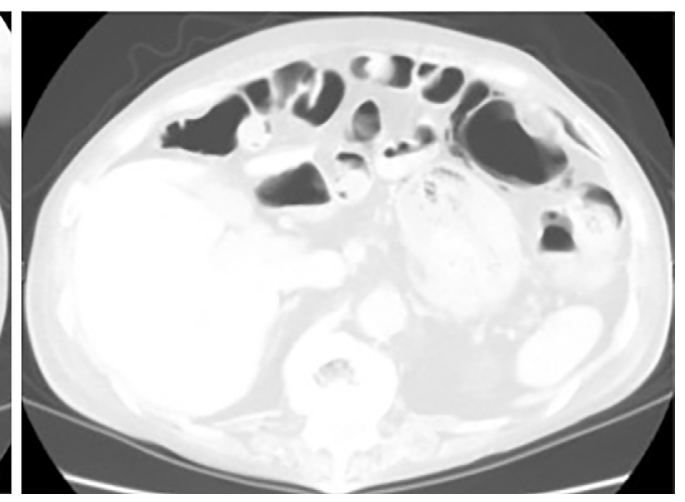

Figure 1. (a) Abdominal computed tomography in Case 2 before treatment for pneumatosis cystoides intestinalis shows free gas in the peritoneum (arrow) and gas in the large intestinal walls. (b) Abdominal computed tomography in Case 2 after treatment of pneumatosis cystoides intestinalis shows no free gas in the peritoneum and improvement of the gas in the large intestinal walls.

kinase (CK), $48 \mathrm{U} / \mathrm{L}$; total cholesterol (TC), $229 \mathrm{mg} / \mathrm{dL}$; blood sugar (BS), $182 \mathrm{mg} / \mathrm{dL}$; HbA1c, 7.9\%; C-reactive protein (CRP), $3.53 \mathrm{mg} / \mathrm{dL}$; and erythrocyte sedimentation rate (ESR), $121 \mathrm{~mm} / \mathrm{h}$. The titers of myeloperoxidase (MPO)ANCA and PR3-ANCA were normal. Chest radiography showed free gas in the peritoneum. The new/worse Birmingham vasculitis activity score (BVAS) was 6 , and the persistent BVAS was 0 at this time. Abdominal computed tomography (CT) showed massive gas accumulation in the small and large intestines and gas in the intestinal wall. PCI was diagnosed based on the clinical signs and images. Sennosides and dimeticone were administered to improve movement in the digestive tract, and voglibose was stopped. The findings on CT were improved one month after starting conservative treatment, and she was discharged.

\section{Case 2}

A 70-year-old woman was admitted to our hospital following a regular visit. Thirty years earlier, she had been diagnosed with RA and treated with methotrexate (MTX). However, interstitial pneumonia was subsequently diagnosed, and administration of MTX was therefore stopped, and she was treated with PSL alone. She had been diagnosed with diabetes mellitus 10 years earlier and was treated with voglibose. At the time of her visit, her body temperature was slightly high. Her height and weight were $152 \mathrm{~cm}$ and $36.9 \mathrm{~kg}$, respectively. The laboratory findings were as follows: WBC count, 17,700/ $\mu \mathrm{L}$ (neutrophils, $88.5 \%$; monocytes, 3.5\%; lymphocytes, 6.5\%); $\mathrm{Hb}, 13.7 \mathrm{~g} / \mathrm{dL}$; platelet count, $22.7 \times 10^{4} / \mu \mathrm{L}$; total protein, $6.3 \mathrm{~g} / \mathrm{dL}$; albumin, $3.1 \mathrm{~g} /$ dL; LDH, $243 \mathrm{U} / \mathrm{L} ; \mathrm{TC}, 178 \mathrm{mg} / \mathrm{dL} ; \mathrm{BS}, 91 \mathrm{mg} / \mathrm{dL} ; \mathrm{HbAlc}$, 5.5\%; CRP, $14.63 \mathrm{mg} / \mathrm{dL}$; and ESR, $38 \mathrm{~mm} / \mathrm{h}$. Her disease activity score 28-erythrocyte sedimentation rate was 4.47 (moderate disease activity), although her visual analogue scale score for pain was always high because of severe back pain. Chest radiography showed massive accumulation of intestinal gas. Abdominal CT showed free gas in the perito- neum and gas in the large intestinal wall (Fig. 1a). PCI was diagnosed. She was administered tosufloxacin tosilate to kill the Gram-negative bacilli in the intestinal tract, and voglibose was stopped. At two weeks after starting treatment, her laboratory and CT findings were improved (Fig. 1b).

\section{Case 3}

A 70-year-old man was admitted to our hospital because of erythema on the face, trunk, and upper extremities and muscle pain in the proximal limbs. Carcinoma of the penis had been diagnosed at 52 years of age, and carcinoma of the skin at 55 years of age, with surgery performed for both. On admission, his height and weight were $156 \mathrm{~cm}$ and 44.2 $\mathrm{kg}$, respectively, and his body temperature was $37.3^{\circ} \mathrm{C}$. A physical examination showed no abnormal auscultation of the chest and no abnormal signs for the abdomen. The laboratory findings were as follows: WBC count, $9,500 / \mu \mathrm{L} ; \mathrm{Hb}$, $13.7 \mathrm{~g} / \mathrm{dL}$; platelet count, $32.0 \times 10^{4} / \mu \mathrm{L}$; total protein, $6.7 \mathrm{~g} /$ $\mathrm{dL}$, albumin, $3.5 \mathrm{~g} / \mathrm{dL}$; aspartate aminotransferase (AST), $227 \mathrm{U} / \mathrm{L}$; alanine aminotransferase (ALT), $102 \mathrm{U} / \mathrm{L}$; LDH, $758 \mathrm{U} / \mathrm{L} ; \mathrm{CK}, 4,665 \mathrm{U} / \mathrm{L} ; \mathrm{TC}, 262 \mathrm{mg} / \mathrm{dL}$; CRP, $0.57 \mathrm{mg} /$ $\mathrm{dL}$; and negative results for anti-Jo-1 antibody. Dermatomyositis was diagnosed and administration of PSL was started at $40 \mathrm{mg} / \mathrm{day}$. However, his ability to swallow worsened, and he could not eat or take medicine. A gastric fistula was therefore created. On hospital day 100, a physical examination showed a distended abdomen. Abdominal X-ray showed massive gas accumulation in the ascending colon, and abdominal CT showed pneumoderma in the abdominal skin on the right side and gas in the ascending colon (Fig. 2). Nutrition via the gastric fistula was stopped, and he was administered high-dose oxygen, kanamycin, and purgative medicines through the gastric fistula. After these treatments were started, the abdominal abnormalities improved. However, the patient died of systemic fungal infection on hospital day 150. No abnormalities, such as gas or fistula, were evident in the small or large intestines on a histologi- 
(a)

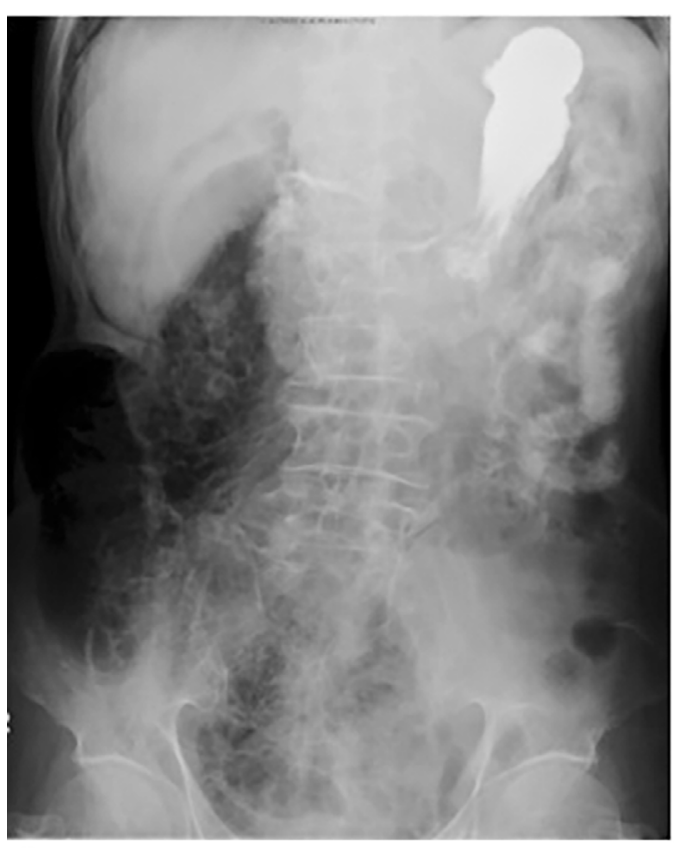

(b)

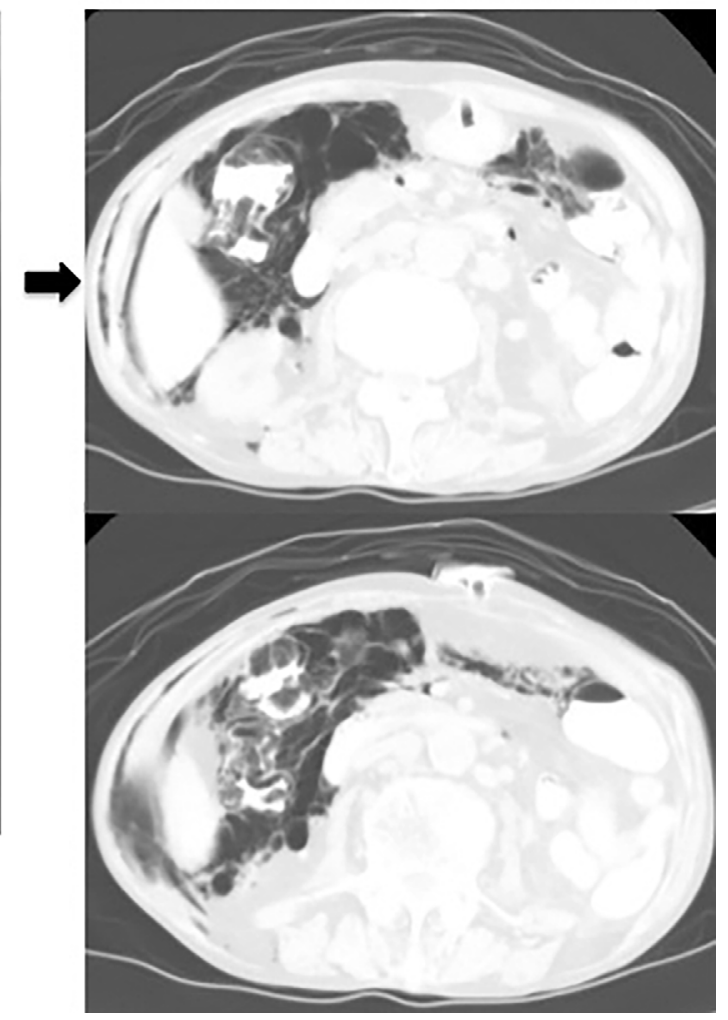

Figure 2. (a) Abdominal X-ray in Case 3 shows massive gas in the ascending colon. (b) Abdominal computed tomography in Case 3 shows pneumoderma in the abdominal skin of the right side (arrow) and gas in the ascending colon.

cal examination at autopsy.

\section{Case 4}

A 42-year-old woman was admitted to our hospital with abdominal distension. Dermatomyositis had been diagnosed based on a skin rash and muscle weakness at a local clinic three years earlier and was treated with PSL and immunosuppressants. However, the disease control was not good. The patient had been diagnosed with ileus half a year before this admission and was being managed conservatively. Her condition initially improved, but she repeatedly experienced feelings of abdominal fullness. A physical examination on admission showed a distended abdomen, reduced bowel sounds, and general tenderness of the abdomen. Furrows were apparent around the mouth perpendicular to the lips, but sclerodactyly was not clear. Her height and weight were $161 \mathrm{~cm}$ and $49 \mathrm{~kg}$, respectively. The laboratory findings were as follows: WBC count, $10,700 / \mu \mathrm{L} ; \mathrm{Hb}, 13.2 \mathrm{~g} / \mathrm{dL}$; platelet count, $19.0 \times 10^{4} / \mu \mathrm{L}$; total protein, $6.2 \mathrm{~g} / \mathrm{dL}$; albumin, 2.7 g/dL; AST, 37 U/L; ALT, 44 U/L; LDH, 324 U/L; CK, $437 \mathrm{U} / \mathrm{L}$; TC, $126 \mathrm{mg} / \mathrm{dL}$; CRP, $5.15 \mathrm{mg} / \mathrm{dL}$; and negative results for anti-Jo-1 antibody, anti-Scl-70 antibody, and anticentromere antibody. Abdominal X-ray showed massive gas accumulation and dilation of the intestines (Fig. 3a). CT showed massive gas accumulation in the intestines and gas in the intestinal walls (Fig. 3b). Scleroderma ( $\mathrm{SSc}$ ) was also diagnosed based on a histological examination of a speci- men from the forearm. Oral ingestion was stopped, and total parenteral nutrition was initiated to allow the gastrointestinal tract to rest. High-dose oxygen was administered, along with purgative medicines. Erythromycin was also administered to promote motilin-responsive movement of the digestive tract. After starting treatment, CT showed reduced findings of gas in the abdomen, and the PCI was improved. The abdominal distension resolved, and the patient was able resume normal eating before discharge.

\section{Discussion}

This report described four different patients with CTD who developed complications with PCI. Vobori reported that $15 \%$ of PCI cases were idiopathic with an unknown etiology, while $85 \%$ were secondary. Various causes of secondary PCI are known, including gastrointestinal disease, obstructive pulmonary disease, and traumatic injury, but CTD is an important base condition, especially in $\operatorname{SSc}(17,18)$. In SSc patients, atrophy and fibrosis of smooth muscle cells in the gastrointestinal walls are observed. Those changes lead to intestinal hypomotility. Bacterial overgrowth and bowel distension are observed in this condition, which can lead to elevated intraluminal pressure and force gas into the intestinal walls $(6,19)$. Bacterial infection has also been proposed to either damage the intestinal wall with subsequent intramural penetration of gas or produce gas, which then pene- 
(a)

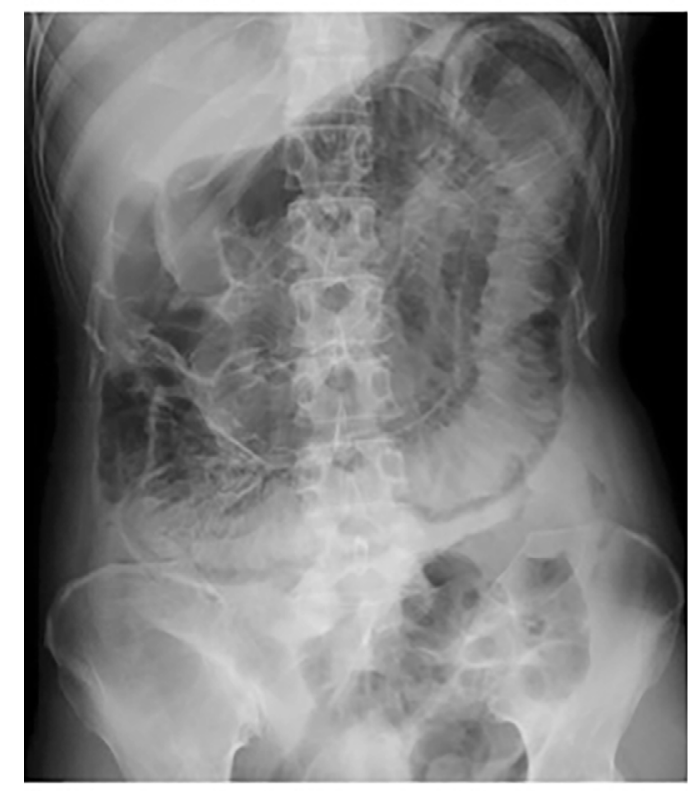

(b)

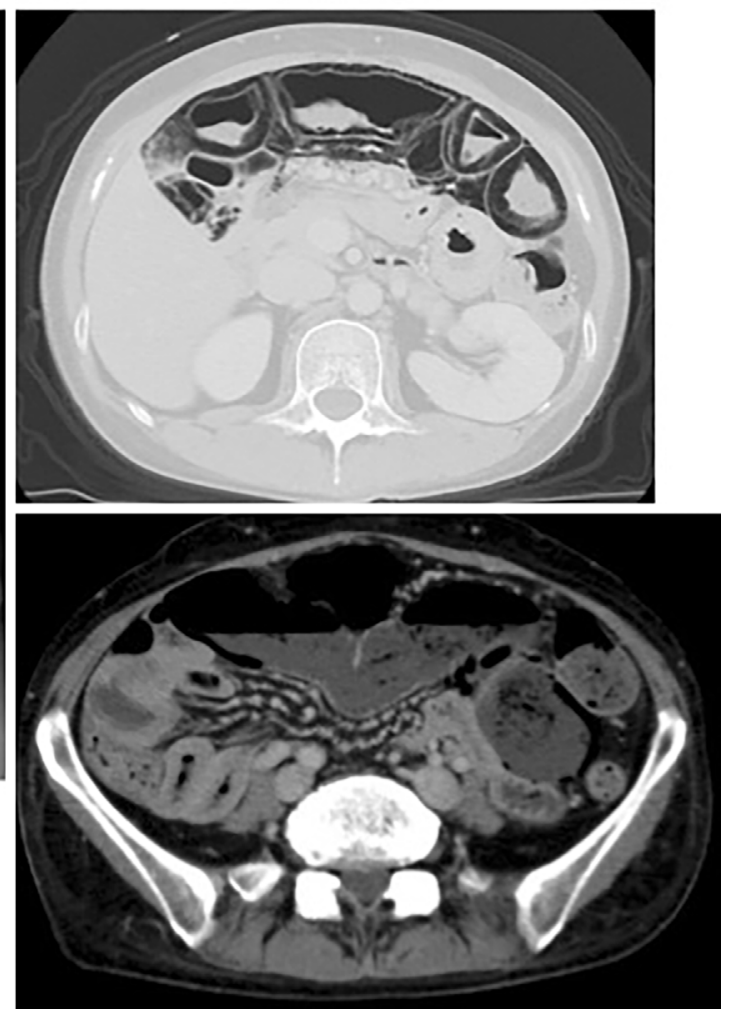

Figure 3. (a) Abdominal X-ray in Case 4 shows massive gas and dilated intestines. (b) Computed tomography in Case 4 shows massive gas in the intestines and gas in the intestinal walls.

trates into the gut wall (15).

As mentioned above, pulmonary disease is also a cause of secondary PCI. Severe coughing can trigger alveolar rupture, which can result in the introduction of air along vascular channels in the mediastinum, tracking downward to the retroperitoneum and then to the mesentery of the bowel. However, the pathogenesis of PCI is not yet fully understood. PCI complicated by systemic lupus erythematosus (SLE) has been reported (2-4). Mizoguchi et al. summarized the findings for 14 SLE patients complicated by PCI in their case report. A pathological analysis was performed in 7 of their cases, with vasculitis was shown in 6. Therefore, unlike SSc, the pathogenesis of PCI complicated by SLE might be vasculitis in the intestines (3).

Some reports have also described polymyositis (PM)/DM patients with PCI. Wada et al. summarized the findings from 14 patients with PM, DM, and overlap syndrome with PCI in their case report. They stated that, among the five reported cases of PCI with juvenile DM, four had high disease activity at PCI onset, and one died of bowel necrosis and perforation, suggesting the occurrence of digestive vasculitis. Thus, subclinical vasculopathy in the bowel mucosa of juvenile DM patients might be related to the occurrence of PCI. However, the disease activity of PM was mostly controlled by the administration of steroids at the time of PCI onset, and the disease activity of adult DM at the onset of PCI was almost the same as those in PM patients with PCI (8). Few cases in which a histological examination was performed have been reported, but the pathogenesis of PCI associated with RA, ANCA-associated vasculitis, and mixed connective tissue disease is hypothesized to involve the existence of microangiopathy in the intestine (10-12).

However, most PCI patients complicated by CTD possess several risk factors for development of the disease. Constipation promotes bacterial overgrowth in the intestines. Bacterial overgrowth and increased intraluminal production of hydrogen may cause a breakdown in mucosal integrity and gas leakage into the bowel wall and peritoneal cavity (18). Several drugs, particularly $\alpha$-GI, have been reported to induce PCI development (13-15). Use of $\alpha$-GI causes flatulence, because absorption of carbohydrates by the colon is suppressed, and intestinal bacteria then generate a large volume of gas through carbohydrate fermentation. This then elevates the intraluminal pressure, allowing the gas-producing bacteria to invade the colonic mucosa through the mucosal breaks, forming pneumocytes. Corticosteroids and immunosuppressants have also been thought to exacerbate the state of PCI. Ammons et al. reported renal transplant patients treated with PSL and cyclosporine A who developed PCI. That report suggested that some medications might shrink Payer's patches, with a resultant loss of structural integrity in the bowel mucosa, allowing for the entry of gas into the bowel wall (20). Corticosteroids and immunosuppressants are often used to treat CTD patients. The administration of steroids and immunosuppressants sometimes induces the onset of diabetes mellitus, and several $\alpha$-GIs have also been 
used to suppress increases in blood glucose levels. CTD patients often show complications of constipation, due to the use of drugs that reduce intestinal movement, and reductions in body movement. These patients are therefore more likely to develop PCI than those without CTD.

In this report, Case 1 was administered corticosteroids to treat GPA, and Case 2 was given a corticosteroid and MTX to treat RA. These two patients showed complications of diabetes mellitus and were treated with voglibose. Because the disease activities of both of these cases were slightly high according, regions affected by vasculitis might have been present in the intestinal walls. Changes to the intestinal wall structure were considered to have been induced by vasculitis in the intestinal walls which originated from GPA or RA, while corticosteroids, and the increased intestinal gas production from the usage of voglibose were considered to be exacerbating factors for PCI development in these two patients. After voglibose was stopped and conservative treatment was implemented, the abdominal conditions improved. Case 3 was administered high-dose corticosteroids to treat active DM but developed dysphagia and subsequently suffered from aspiration pneumonia. He stopped eating and drinking but then developed severe constipation. Although we were unable to confirm the existence of vasculitis in the intestinal wall in the autopsy examination, structural changes in the intestinal wall caused by vasculitis might have been related to PCI development because his dermatomyositis was active during the clinical course. High-dose prednisolone might deplete lymphoid tissue with resulting mucosal disruption, severe constipation-induced bacterial overgrowth, and high pressure in the intestine. Malnutrition and aspiration pneumonia might influence intestinal mobility, bacterial growth in the intestine, and permeability of the intestinal wall. Case 4 showed proliferation of collagenous fibers on a skin biopsy. She developed repeated intestinal pseudoobstruction half a year before she developed PCI. Fibrosis of the intestinal wall was thus suggested to have progressed rapidly. This patient may have been predisposed to PCI due to a combination of pathologies such as microangiopathy, atrophy and fibrosis of the intestinal layers, impaired intestinal motility, bacterial overgrowth, elevated intraluminal pressure, and increased permeability of the intestinal wall. Case 4 had been treated with corticosteroids and immunosuppressants for several years due to refractory dermatomyositis. The usage of those immunosuppressive agents was thus also considered to have contributed to the development of PCI. She also developed interstitial pneumonia as a complication, but did not complain of dyspnea during the clinical course. The interstitial pneumonia was thus considered unrelated to the occurrence of PCI in this patient. In all four patients, development of PCI was attributed to CTD plus several aggravating factors.

In conclusion, PCI is a rare disease, but various CTDs, particularly SSc and SLE, appear to represent important underlying diseases causing secondary PCI. Although immunosuppressants and CTDs are notable risk factors for the oc- currence of PCI, many CTD patients receive these agents to improve their condition. CTD patients must therefore be carefully observed for abdominal symptoms, and in patients complicated with diabetes mellitus or constipation, we must pay attention to their condition and provide treatment as soon as possible before the complications become exacerbated.

The authors state that they have no Conflict of Interest (COI).

\section{References}

1. Sagara A, Kitagawa K, Furuichi K, et al. Three cases of pneumatosis intestinalis presenting in autoimmune disease. Mod Rheumatol 22: 610-615, 2012.

2. Yamaguchi Y, Ohno S, Yamazaki S, et al. A case of systemic lupus erythematosus complicated with pneumatosis cystoides intestinalis. Mod Rheumatol 15: 440-444, 2005.

3. Mizoguchi F, Nanki T, Miyasaka N. Pneumatosis cystoides intestinalis following lupus enteritis and peritonitis. Intern Med 47: 1267-1271, 2008.

4. Shimojima Y, Ishii W, Matsuda M, Tojo K, Watanabe R, Ikeda S. Pneumatosis cystoides intestinalis in neuropsychiatric systemic lupus erythematosus with diabetes mellitus: case report and literature review. Mod Rheumatol 21: 415-419, 2011.

5. Quirouz ES, Flannery MT, Martinez EJ, Warner EA. Pneumatosis cystoides intestinalis in progressive systemic sclerosis: a case report and literature review. Am J Med Sci 310: 252-255, 1995.

6. Honne K, Maruyama A, Onishi S, Nagashima T, Minota S. Simultaneous pneumatosis cystoides intestinalis and pneumomediastinum in a patient with systemic sclerosis. J Rheumatol 37: 21942195, 2010.

7. Wilson FC, Warrington KJ. Pneumatosis intestinalis in limited scleroderma. J Rheumatol 35: 352-353, 2008.

8. Wada Y, Murayama N, Hirose S, et al. A case of pneumatosis cystoides intestinalis in a patient with polymyositis and interstitial pneumonia. Mod Rheumatol 14: 260-263, 2004.

9. Zarbalian Y, von Rosenvinge EC, Twadell W, Mikdashi J. Recurrent pneumatosis intestinalis in a patient with dermatomyositis. BMJ Case Rep 2013: bcr2013200308, 2013.

10. Aoki $Y$, Nagashima $T$, Kamimura $T$, Iwamoto $M$, Minota $S$. Marked pneumatosis cystoides intestinalis in a patient with mixed connective tissue disease. J Rheumatol 33: 1705-1706, 2006.

11. Nakagawa $S$, Akimoto $T$, Takeda $S$, et al. Antineutrophil cytoplasmic antibody-associated glomerulonephritis complicated by pneumatosis intestinalis. Clin Med Insights Case Rep 8: 65-70, 2015.

12. Caroline CY, Marzan KA. Benign pneumatosis intestinalis in a pediatric patient with multiple risk factors including granulomatosis with polyangiitis: a case report and review of the literature. Semin Arthtitis Rheum 44: 423-427, 2015.

13. Hisamoto A, Mizushima T, Sato K, et al. Pneumatosis cystoides intestinalis after alpha-glucosidase inhibitor treatment in a patient with interstitial pneumonitis. Intern Med 45: 73-76, 2006.

14. Tsujimoto T, Shioyama E, Moriya K, et al. Pneumatosis cystoides intestinalis following alpha-glucosidase inhibitor treatment: a case report and review of the literature. World J Gastroenterol 14: 6087-6092, 2008.

15. Kojima K, Tsujimoto T, Fujii H, et al. Pneumatosis cystoides intestinalis induced by the $\alpha$-glucosidase inhibitor miglitol. Intern Med 49: 1545-1548, 2010.

16. Han BG, Lee JM, Yang JW, Kim MS, Choi SO. Pneumatosis intestinalis associated with immune-suppressive agents in a case of minimal change disease. Yonsei Med J 43: 686-689, 2002.

17. Voboril R. Pneumatosis cystoides intestinalis: a review. Acta 
Medica (Hradec Kralove) 44: 89-92, 2001.

18. Balbir-Gurman A, Brock OR, Chermesh I, Braun-Moscovici Y. Pneumatosis cystoides intestinalis in scleroderma-related conditions. Intern Med J 42: 323-329, 2012.

19. Young MA, Rose S, Reynolds JC. Gastrointestinal manifestations of scleroderma. Rheum Dis Clin North Am 22: 797-823, 1996.

20. Ammons MA, Bauling PC, Weil R 3rd. Pneumatosis cystoides intestinalis with pneumoperitoneum in renal transplant patients on cyclosporine and prednisone. Transplant Proc 18: 1868-1870, 1986.

The Internal Medicine is an Open Access article distributed under the Creative Commons Attribution-NonCommercial-NoDerivatives 4.0 International License. To view the details of this license, please visit (https://creativecommons.org/licenses/ by-nc-nd/4.0/).

(C) 2017 The Japanese Society of Internal Medicine http://www.naika.or.jp/imonline/index.html 